\title{
Sur la présence d'Andrena binominata SMITH, 1853 (Hymenoptera, Andrenidae) et de Nomada duplex SmITH, 1854 (Hymenoptera, Apidae) en France
}

\author{
Par Matthieu Aubert * , Eric Dufrêne **, David GenOud ***, Erwin SCHEUCHL ${ }^{4 *}$ et Maximilian SCHWARZ ${ }^{5 *}$
}

\begin{abstract}
The bees Andrena (Carandrena) binominata SMITH, 1853 and his supposed cuckoo Nomada duplex SMITH, 1854 are reported from the surroundings of Perpignan (Pyrénées-Orientales, France) for the first time and some observations made on the field in 2010 are presented. Furthermore, the geographic distribution of both species is synthesized and illustrated with a map. The new data are also discussed with an emphasis on conservation biology.
\end{abstract}

Résumé. La découverte d'Andrena (Carandrena) binominata SMITH, 1853 ainsi que celle de son parasite présumé Nomada duplex Smith, 1854 en France à proximité de Perpignan (Pyrénées-Orientales) en 2009 et des observations réalisées à cette occasion et en 2010 sont présentées. Les connaissances sur la répartition des deux espèces sont ensuite synthétisées et reprises sur une carte. Enfin, les nouvelles données sont discutées et des éléments de biologie de la conservation mis en avant.

Mots-clés. Andrena binominata, Nomada duplex, France.

\section{Introduction}

Les genres Andrena FABRICIUs, 1775 et Nomada SCOPOLI, 1770 comptent à eux seuls plus d'un quart des espèces d'abeilles de la faune française. Selon la liste taxonomique des abeilles de France, de Belgique, de Suisse et du Grand Duché du Luxembourg (Rasmont et al. 1995), le premier en rassemble 153 et le second 83 sur un total de 865. En l'état de nos connaissances, près de 160 espèces d'Andrènes et près de 90 espèces de Nomades sont connues de France métropolitaine, Corse incluse, pour un nombre d'espèces d'abeilles total peutêtre supérieur à 900 .

Les Andrènes sont des abeilles dites solitaires, par opposition à celles sociales que sont l'Abeille mellifère (Apis mellifera LinNé 1778) et les Bourdons (Bombus spp.). Toutes nichent dans le sol, une grande part ayant une préférence marquée pour les substrats sablonneux. La majorité des espèces est active au printemps et beaucoup sont oligolectiques c'est-à-dire qu'elles sont spécialisées pour la récolte du pollen et l'alimentation des larves sur un spectre de plantes relativement étroit -

* Matthieu Aubert, 7 rue du Maréchal Fayolle, F-13004 Marseille, France. E-mail: matthieuaubert@gmail.com

** Eric Dufrêne, Ecologie Systématique \& Evolution (ESE, UMR8079, GNRS \& Univ. Paris-Sud Orsay, AgroParisTech), Université Paris-Sud XI, Bât. 362, F-91405 Orsay Cedex, France.

E-mail: eric.dufrene@u-psud.fr

*** David Genoud, 10 Rue du Président Fallières, F-11000 Carcassonne, France. E-mail: dge-davidgenoud@orange.fr

4* Erwin Scheuchl, Kastanienweg 19, D-84030 Ergolding, Allemagne. E-mail: erwin.scheuchl@t-online.de

5* Maximilian Schwarz, Eibenweg 6, A-4052 Ansfelden, Autriche. Email: maximilian.schwarz@liwest.at une famille, un genre ou plus rarement une espèce particulière (Westrich 1989; Michener 2007).

Les Nomades sont quant à elles des abeilles qui vivent aux dépens d'autres abeilles. Elles appartiennent au groupe des espèces cleptoparasites et sont vulgairement appellées « abeilles-coucou » par analogie avec l'oiseau. Comme chez celui-ci, leur progéniture prend la place de celle de l'hôte dans le nid et accapare les ressources alimentaires. Lorsqu'une femelle Nomade entre dans le nid d'une espèce hôte qui lui convient, cette première peut détruire directement l'œuf en place mais le plus souvent elle se contente de pondre et c'est la larve du parasite qui détruit celle de l'hôte. La larve du parasite utilise ensuite les provisions de pollen et de nectar emmagasinées par les femelles de l'espèce hôte pour assurer son développement. Contrairement à d'autres genres cleptoparasites comme les Sphecodes, une espèce de Nomade parasite le plus souvent une seule espèce (plus rarement quelques espèces) avec une nette préférence pour les Andrènes. Toutefois, certaines parasitent des espèces des genres Panurgus, Melitta, Eucera et Lasioglossum (Westrich 1989; Michener 2007).

\section{Observations}

A l'occasion d'un séjour dans les PyrénéesOrientales au sortir de l'hiver 2009, les 26 et 27 février, l'un d'entre nous (MA) a profité de belles journées ensoleillées pour sortir le filet à papillons à la recherche d'abeilles et autres aculéates. Les quelques prospections entreprises ont été réalisées non loin de Perpignan, sur la commune de Pia (WGS84 42 $45{ }^{\prime} 25^{\prime}$ N 2 ${ }^{\circ} 55^{\prime} 16$ 'E), en milieu périurbain au sein principalement de friches 

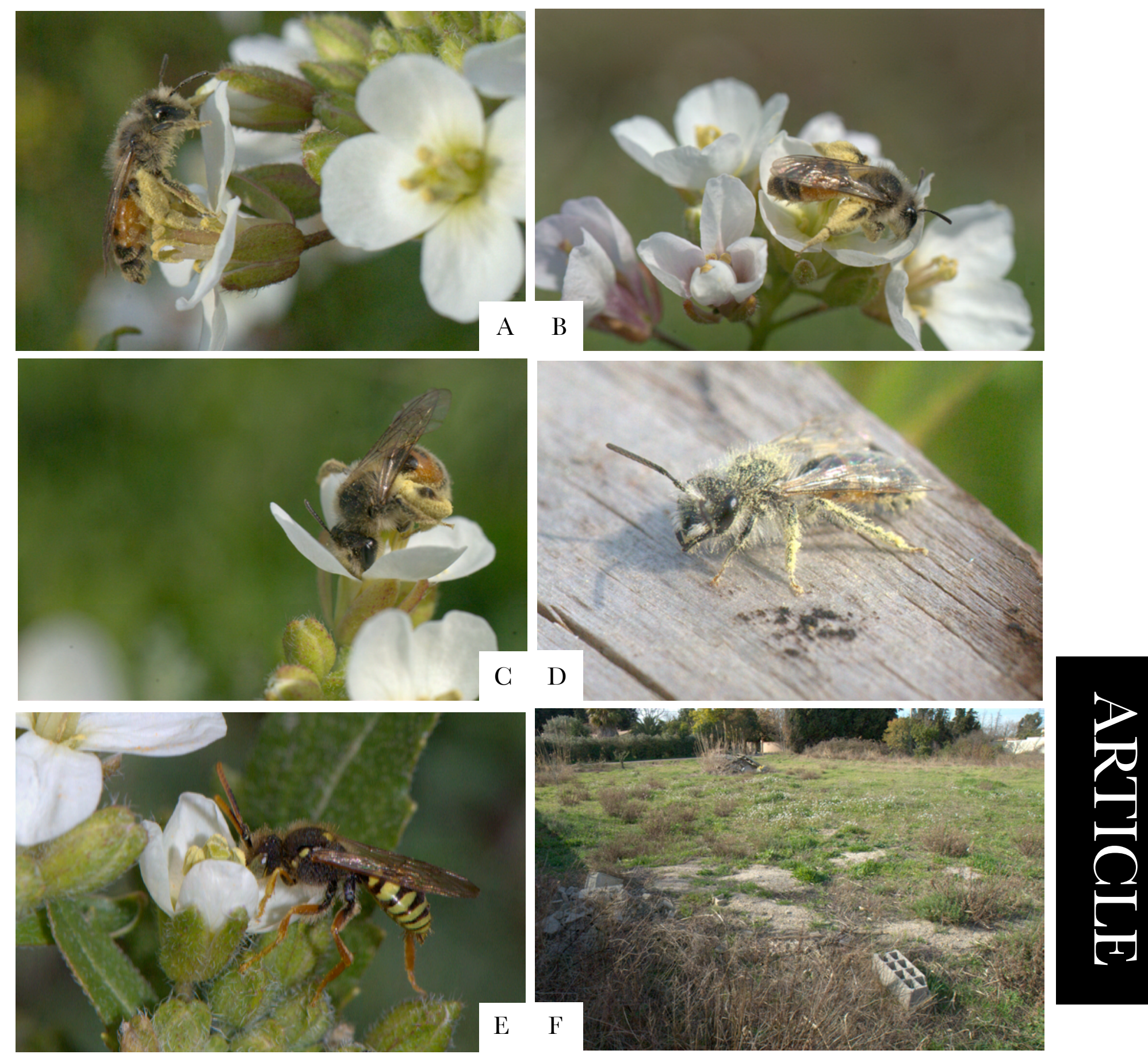

Figure 1. A-C: Andrena (Carandrena) binominata SMITH (Hym. Andrenidae) femelle sur Diplotaxis erucoides (L.) (Brassicaceae); D: mâle d'A. (C.) binominata au repos; E: Femelle de Nomada duplex SMITH (Hym. Apidae) sur D. erucoïdes; F: vue du site où les observations décrites dans cet article ont été réalisées (Photos M Aubert sauf $\mathbf{E}$ par D Genoud)

où fleurissaient le Diplotaxis fausse-roquette (Diplotaxis erucoüdes (L.)) et le Souci des champs (Calendula arvensis L.), pour les espèces les plus visibles. La douceur de la météo a permis notamment la récolte de plusieurs abeilles dont des Andrènes (Andrena spp.) et une Nomade (Nomada sp.) (Tableau 1).

Leur détermination ultérieure a révélé notamment la présence à Pia d'Andrena binominata SMITH, 1853 et Nomada duplex SMITH, 1854. Nous avons alors recherché des informations sur ces espèces peu communes dans la bibliographie et auprès de plusieurs collègues en Europe. Nous nous sommes rendus compte assez rapidement que nous étions en présence de la première donnée de $\mathcal{N}$. duplex pour la France. En ce qui concerne
A. binominata en revanche, nous l'avons trouvée mentionnée en France sur le site internet de l'INPN (http:/inpn.mnhn.fr). Des contacts auprès de cet organisme (Arnaud Hourellou et Olivier Gargominy) nous ont signalé que ces données provenaient du transfert des informations du référentiel Fauna Europaea (www.faunaeur.org), où l'espèce est effectivement cartographiée pour la France. Ce n'est pas le cas cependant dans la liste taxonomique des abeilles de France (Rasmont et al. 1995), où elle est seulement indiquée comme étant présente en Espagne, ni dans le catalogue des Andrènes du monde (Gusenleitner \& Schwarz 2002). Nous avons alors recherché l'origine de l'information présentée sur le site internet. Malgré nos efforts, nous n'avons pu l'obtenir et 
pour cause, il semble bien que ce soit une erreur et que notre donnée de Pia représente une première pour $A$. binominata en France également. Nous présentons plus avant dans l'article une synthèse des données relatives à cette espèce, disponibles jusqu'alors dans la bibliographie.

Entre temps, avant d'avoir pu établir toute certitude, un retour à Pia a été programmé au début du mois de mars 2010 dans l'espoir de revoir les deux espèces mais aussi d'observer la femelle de $\mathcal{N}$. duplex et de trouver les nids d'A. binominata pour essayer de préciser leur relation. Nous avons alors bien retrouvé $A$. binominata et des mâles de son parasite présumé, mais toujours pas de femelle, ni de nid.

Déjà soupçonné après les visites de 2009, la préférence marquée d'A. binominata pour le Diplotaxis pour la récolte du pollen a été confortée par l'observation récurrente de femelles en train d'en prélever sur cette crucifère (Figure 1A-G). Les femelles de cette espèce n'ont pas été vues par ailleurs, si ce n'est en contrebas ou non loin de pieds de la plante sur le feuillage de la végétation herbacée. Quant aux mâles, ils ont pu être vus patrouillant aux abords des massifs de Diplotaxis fausse-roquette. Un accouplement furtif (ou peut-être seulement une tentative) a pu être observé : de manière classique, un mâle s'est jeté sur une femelle affairée à ramasser du pollen sur une fleur. Les deux partenaires ont rapidement chuté au sol et la femelle s'est alors extirpé de l'étreinte du mâle sans grand délai. Le mâle resté seul a marqué un temps d'arrêt dans son inlassable agitation et a pu être photographié (Figure 1D) à cette occasion.

En ce qui concerne les mâles de $\mathcal{N}$. duplex, ils ont été principalement observés en train de se restaurer sur le Souci des champs et le Diplotaxis. Ils ont été capturés aussi alors qu'ils patrouillaient à la surface du sol. Notons qu'en 2010, un autre secteur de la commune a été prospecté et que des mâles de cette espèce y ont été attrapés. Cela n'a pas été le cas pour $A$. binominata.

Malgré des recherches en ce sens, aucun nid de cette espèce n'a pu être trouvé. Il est probable que ceux-ci soient dissimulés sous la végétation herbacée et que les éventuelles agrégations soient lâches, rendant les observations difficiles. De même la femelle de $\mathcal{N}$. duplex n'a pu être découverte ce qui laisse planer un doute légitime sur la relation de parasitisme entre les deux espèces. Celle-ci n'est en outre pas documentée dans la bibliographie. Cependant, la présence simultanée sur un même site, deux années consécutives de ces deux espèces nouvelles pour la France nous amène à poser l'hypothèse qu'il existe une relation de parasitisme entre elles, d'autant plus que celles-ci présentent une distribution similaire et ont été découvertes plusieurs fois dans les mêmes régions (Figure 2). Le cas échéant, on pourrait se poser également la question de savoir si $\mathcal{N}$. duplex serait liée à d'autres espèces hôtes. Cette relation, exclusive ou non, devra être confirmée. D'autres espèces d'Andrènes et une Eucère (voir Tableau 1), susceptibles d'êtres des hôtes pour Nomada duplex, ont été recensées sur le site à la même période ce qui nous amènent également à être prudents.

L'ensemble de nos visites printanières nous ont permis d'inventorier huit espèces d'andrènes (Andrena spp.), une nomade ( $\mathcal{N}$. duplex), une lasioglosse (Lasioglossum malacharum), en plus de l'observation d'ouvrières de l'Abeille mellifère, de reines et quelques (premières) ouvrières du Bourdon terrestre (Bombus terrestris), d'un mâle d'Eucère (Eucera nigrilabris) et d'un mâle d'Anthophore (Anthophara plumipes). Le détail des taxons contactés est repris au Tableau 1.

\section{Synthèse des données biogéographiques relatives à Andrena binominata SMITH, 1853}

Andrena binominata présente une distribution ouestméditerranéenne. Elle est connue d'Italie (Sicile), de Malte, de Tunisie (Médénine, région de Tunis), d'Algérie (région d'Oran), du Maroc (région de Marrakech) et d'Espagne (Baléares, régions de Valence, de Madrid et de Barcelone). Son aire de répartition est relativement vaste mais sa distribution apparaît morcelée. En tout, seulement une quinzaine de localités est mentionnée dans la bibliographie, dont plus de la moitié en Espagne (Bofill y Pichot 1905 ; Friese, 1922 ; Gusenleitner \& Schwarz, 2002 ; Lepeletier, 1841 ; Lucas, 1849 ; Pérez, 1903 ; de Stefani, 1882 ; Warncke, 1967, 1974, 1976). L'espèce y est parfois citée sous des noms différents : A. bimaculata LEPELETIER, 1841, $A$. griseofusca PÉREZ, 1903 et Andrena mallorcana FRIESE, 1922.

\section{Synthèse des données biogéographiques relatives à Nomada duplex SMITH, 1854}

La distribution de $\mathcal{N}$. duplex est similaire à celle d'A. binominata, de type ouest-méditerranéenne. Cette

Table 1. Synthèse des captures d'abeilles sauvages sur le site d'étude à Pia (Pyrénées-Orientales, France)

\begin{tabular}{|l|l|l|l|}
\hline \multirow{5}{*}{ Andreninae } & Andrena binominata & Andreninae & Andrena rhenana \\
\cline { 2 - 3 } & Andrena fulva & Halictinae & Lasioglossum malacharum \\
\cline { 2 - 2 } & Andrena lagopus & & Nomada duplex \\
\cline { 2 - 2 } & Andrena nigroaenea & Anthophora plumipes \\
\cline { 2 - 3 } & Andrena nitida & & Eucera nigrilabris \\
\cline { 2 - 3 } & Andrena (Micrandrena) sp. & Bombus terrestris \\
\cline { 2 - 4 } & Andrena rosae & Apis mellifera \\
\hline
\end{tabular}






Figure 2. Synthèse des données biogéographiques relatives à Andrena (Carandrena) binominata SMITH (Hym. Andrenidae) et à Nomada duplex SMITH (Hym. Apidae)

Nomade est présente en Algérie (région d'Alger, Annaba, Biskra, Constantine, Oran, Mascara et SidiBel-Abbès), en Espagne (Séville, région de Cordoue, Almeria, régions de Ciudad Real et de Madrid, Saragosse, Barcelone), en Italie (Ancona, Pescora, Sardaigne, Sicile), en Libye, au Maroc (Essaouira) et en Tunisie (Tunis). Comme A. binominata, c'est dans la péninsule ibérique que les données de $\mathcal{N}$. duplex sont les plus nombreuses. Un nombre légèrement plus grand de localités est connu pour cette espèce (Dusmet y Alonso 1913, 1915; Gribodo in Schwarz 1981; Ortiz-Sanchez 1992, coll. pers. ; Schwarz 1977, coll. pers.; Saunders 1908). Jusqu'à récemment, $\mathcal{N}$. duplex SMITH, 1854 était confondue avec $\mathcal{N}$. cirtana PÉREZ, 1895, parfois avec $\mathcal{N}$. fucata melanoscopa GRIBODO 1894 et $\mathcal{N}$. fucata nigroflavida GRIBODO, 1894.

\section{Discussion}

La position des Pyrénées-Orientales sur le littoral méditerranéen et limitrophe de l'Espagne ainsi que sa grande variété de milieux lié en partie à un gradient altitudinal très important, en font l'un des départements de France et l'une des régions d'Europe où la biodiversité est la plus riche. Cela a attiré depuis longtemps les naturalistes dont notamment des apidologues (Rasmont 1998 ; Michez et al. 2004). La grande majorité des recherches se sont focalisées logiquement sur des secteurs plus ou moins naturels. Ceux soumis plus fortement à la pression anthropique comme la plaine de Perpignan où a eu lieu l'heureuse découverte d'A. binominata et de $\mathcal{N}$. duplex ont été bien moins prospectés. De plus, la grande précocité de leur période d'activité, centrée sur la fin février, rendait leur découverte plus hasardeuse. Ainsi, même si on ne peut exclure totalement une colonisation récente, il est probable que ces deux espèces soient présentes dans la région depuis une longue durée et simplement passées inaperçues.

Leur découverte s'est faite au sein d'habitats rudéraux, des friches péri-urbaines en l'occurrence. Ce type d'habitat n'étant pas spécialement rare dans la région de Perpignan, $A$. binominata et $\mathcal{N}$. duplex, bien que localisées, pourraient y être relativement communes. La prospection d'une autre zone en 2010, toujours sur la commune de Pia mais de l'autre côté de l'agglomération, à plus d'un $\mathrm{km}$ à vol d'oiseau du premier site, a d'ailleurs permis la capture de mâles de $\mathcal{N}$. duplex. Le Diplotaxis fausse-roquette est en outre une plante très commune dans les friches et les vignes du sud de la France.

En dépit du caractère anthropisé des sites d'observations, on peut raisonnablement penser que l'étalement urbain et surtout la densification de l'habitat représentent des menaces importantes pour ces deux espèces d'abeilles. Dans les contextes urbains et périurbains, la conservation d'une mosaïque d'habitats tels que parcelles agricoles (non soumises à une pression chimique trop intense), friches voire espaces verts (gérés de manière différenciée), permet le maintien d'un certain niveau de biodiversité dite ordinaire. Dans le cas présent, elle assurerait sans doute aussi la pérennité de 
ces deux espèces remarquables pour la faune française. L'amélioration des connaissances sur leur répartition et la localisation des sites de nidifications sont primordiales dans cette perspective.

\section{Remerciements}

Nous tenons à remercier tous ceux-ci qui nous donné des indications pour retrouver l'origine de la mention sur Fauna Europea d'Andrena binominata en France, à savoir Messieurs Fritz Gusenleiner, Hans Schwenninger, Quentin Rome, Arnaud Hourellou et Olivier Gargominy. Merci également à Javier Ortiz-Sanchez qui nous a transmis des informations sur la répartition de Nomada duplex en Espagne. Enfin, mille merci à Jessica Gross et Azdin Moumou pour leur hospitalité.

\section{Références bibliographiques}

Bofill y Pichot JM, 1905. Catàlech de insectes de Catalunya, Hymenòpters, XIX. Familia Apidae. Institució Catalana d'Història Natural 22, Barcelona, 74pp.

Dusmet y Alonso JM, 1913. Los Apidos de Espana IV. Gen. Nomada FABR. Memorias de la Real Sociedad Española de Historia Natural 9: 203-395

Friese H, 1922. Neue Formen der Bienengattung Andrena. Konowia 1 (4-5): 209-217.

Gusenleitner F \& Schwarz M, 2002. Weltweite Checkliste der Bienengattung Andrena mit Bemerkungen und Ergänzungen zu paläarktischen Arten (Hymenoptera, Apidae, Andreninae, Andrena). Entomofauna suppl. 12: 1-1280.

Lepeletier A, 1841. Histoire naturelle des Insectes. Hyménoptères. 2: 248-249

Lucas H, 1849. Exploration scientifique de l'Algérie pendant les années 1840, 1841, 1842. Sciences physiques - Zoologie. Dans Histoire naturelle des animaux articulés, Troisième partie - Insectes. Imprimerie Nationale, Paris.

Michener CD, 2007. The bees of the world. Second Edition. The John Hopkins Univesity Press, Baltimore.
Michez D, Patiny S \& Iserbyt S, 2004. Apoidea remarquables observés dans les Pyrénées-Orientales, France (Hymenoptera, Andrenidae, Melittidae). Bulletin de la Société Entomologique de France 109 (4) : 379-382

Ortiz-Sánchez FJ, 1992. Bees from the province of Almería, additional records (Hymenoptera, Apoidea). Boletim Sociedade Portuguesa Entomologia (Suplemento) 3: 623-632.

Pérez J, 1895. Espèces nouvelles de Mellifères de Barbarie (Diagnoses préliminaires). G. Gounouilhou, Bordeaux, 65 p.

Pérez J, 1903. Espèces nouvelles de Mellifères. Procès-verbaux des séances de la Société Linnéenne de Bordeaux 58: 78-93.

Rasmont P, Ebmer AW, Banazsak J, Van der Zanden G, 1995. Hymenoptera Apoidea Gallica. Liste taxonomique des abeilles de France, de Belgique, de Suisse et du Grand-Duché de Luxembourg. Bulletin de la Société Entomologique de France (hors série) 100 : 1-98.

Rasmont P, 1998. Rapport préliminaire sur la faune des bourdons (Hymenoptera, Bombinae) des P-O. R.N. Massane et vallon d'Eyne. Travaux de la Réserve Naturelle de la Massane, Banyuls- sur-Mer 52: 1-17.

Saunders E. 1908. Hymenoptera Aculeata collected in Algeria. Part III - Anthophila. Transactions of the Entomological Society of London 2: 177-273.

Schwarz M, 1977. Ergebnisse der Untersuchungen der von J.Pérez 1895 in "Espèces nouvelles de Méllifères de Barbarie" beschriebenen Nomada-Arten und Beschreibung von vier neuen Arten (Hymenoptera, Apoidea). Mitteilungen der Münchner entomologischen Gesellschaft 66: 39-79.

Schwarz M, 1981. Zur Kenntnis des von Gribodo (1894) beschriebenen und behandelten Nomada-Arten (Hymenoptera, Apoidea). Entomofauna 2(5) : 57-75.

de Stefani T, 1882. Imenotteri nuovi opoco conosciuti della Sicilia. Naturalista Siciliano 1 (1881-82): 156.

Warncke K, 1967. Faunistische Bemerkungen über westpaläarktische Bienen der Gattung Andrena F. Bulletin des Recherches agronomiques de Gembloux 2(3): 569-581.

Warncke K, 1974. Beitrag zur Kenntnis und Verbreitung der Sandbienen in Nordafrika. Mitteilungen aus dem Zoologischen Museum in Berlin 50(1): 1-53.

Warncke K, 1976. Die Bienengattung Andrena F., 1775, in Iberien. Teil B. Eos 50 [1974]: 119-223.

Westrich P, 1989. Die Wildbienen Baden-Württembergs. Ulmer Verlag Stuttgart, Band I und II. 